\title{
Transfusão de Sangue em Terapia Intensiva: Um Estudo Epidemiológico Observacional ${ }^{\star}$
}

\author{
Blood Transfusion in Intensive Care: \\ An Epidemiological Observational Study
}

José Rodolfo Rocco ${ }^{1}$, Márcio Soares ${ }^{2}$, Rodolfo Andrade Espinoza ${ }^{3}$

\section{RESUMO}

JUSTIFICATIVA E OBJETIVOS: A transfusão de concentrado de hemácias ( $\mathrm{CHA}$ ) é muito freqüente no centro de tratamento intensivo (CTI), mas as conseqüências da anemia nos pacientes gravemente enfermos ainda são obscuras. Os objetivos desse estudo foram avaliar a freqüência, as indicações, os limiares transfusionais e o prognóstico dos pacientes criticamente enfermos que receberam $\mathrm{CHA}$.

MÉTODO: Estudo prospectivo de coorte realizado no CTI médico-cirúrgico de um Hospital Universitário durante 16 meses. Foram coletados dados demográficos, clínicos e os relacionados a transfusão de CHA.

1. Médico do Centro de Tratamento Intensivo do Hospital Universitário Clementino Fraga Filho da UFRJ, Professor Adjunto do Departamento de Clínica Médica da FMUFRJ, Mestre e Doutor em Clínica Médica pela UFRJ, Título de Especialista em Terapia Intensiva pela Associação de Medicina Intensiva Brasileira e pela Federação Pan Americana e Ibérica de Medicina e Cuidados Intensivos, Titular-Colaborador do Colégio Brasileiro de Cirurgiões.

2. Médico do Centro de Tratamento Intensivo do Hospital I do Instituto Nacional de Câncer (INCA), Mestre e Doutor em Clínica Médica pela UFRJ, Título de Especialista em Terapia Intensiva pela Associação de Medicina Intensiva Brasileira,

3. Médico da Rotina do Centro de Tratamento Intensivo Cirúrgico do Hospital Copa D'Or. Ex-Residente de Terapia Intensiva do Hospital Universitário Clementino Fraga Filho da UFRJ.

*Recebido do Centro de Tratamento Intensivo do Hospital Universitário Clementino Fraga Filho da Universidade Federal do Rio de Janeiro (UFRJ), Rio de Janeiro, RJ

Apresentado em 11 de julho de 2006

Aceito para publicação em 28 de agosto de 2006

Endereço para correspondência:

Dr. José Rodolfo Rocco

Rua Desenhista Luiz Guimarães, 70 Bloco 1/602

Barra da Tijuca

22793-261 Rio de Janeiro, RJ

Fone (21) 2431-7301 Fax. (21) 3325-4579

E-mail: jrrocco@globo.com

(C)Associação de Medicina Intensiva Brasileira, 2006
Regressão logística binária foi utilizada após as análises univariadas.

RESULTADOS: Dos 698 pacientes internados, 244 (35\%) foram transfundidos com CHA. Os pacientes clínicos e em pós-operatório de urgência foram mais transfundidos. Os limiares transfusionais foram: hematócrito $=22,8 \% \pm 4,5 \%$ e hemoglobina $=7,9 \pm 1,4$ $\mathrm{g} / \mathrm{dL}$. Os pacientes transfundidos receberam em média 4,4 \pm 3,7 CHA e apresentaram maior letalidade no CTI $(39,8 \%$ versus $13,2 \% ; p<0,0001)$ e no hospital $(48,8 \%$ versus $20,3 \% ; p<0,0001)$. A letalidade correlacionouse com o número de CHA transfundidos $\left(R^{2}=0,91\right)$. $\mathrm{Na}$ análise multivariada, os fatores relacionados com a necessidade de transfusão foram cirrose hepática, ventilação mecânica (VM), tipo e duração da internação no CTI, hematócrito e escore SAPS II. Os fatores independentes relacionados à letalidade hospitalar foram: VM, número de transfusões de $\mathrm{CHA}>5$ unidades e escore SAPS II.

CONCLUSÕES: A transfusão de $\mathrm{CHA}$ é freqüente no CTI, particularmente nos pacientes internados por problemas clínicos e após cirurgias de emergência, com internação prolongada, em VM e com cirrose hepática. O limiar transfusional observado foi mais baixo que aquele assinalado pela literatura. A transfusão de $\mathrm{CHA}$ foi associada com maior letalidade.

Unitermos: Concentrado de Hemácias, Prognóstico, Terapia Intensiva, Transfusão de sangue

\section{SUMMARY}

BACKGROUND AND OBJECTIVES: Packed red blood cell (PRBC) transfusion is frequent in intensive care unit (ICU). However, the consequences of anemia in ICU patients are poorly understood. Our aim was to evaluate the prevalence, indications, pre-transfusion hematocrit and hemoglobin levels, and outcomes of ICU patients transfused with PRBC.

METHODS: Prospective cohort study conducted at a 
medical-surgical ICU of a teaching hospital during a 16-month period. Patients' demographic, clinical, laboratory and transfusion-related data were collected. Logistic regression was used after univariate analyses. RESULTS: A total of 698 patients were evaluated and 244 (35\%) received PRBC, mainly within the first four days of ICU (82.4\%). Transfusion was more frequent in medical and emergency surgical patients. The mean pre-transfusion hematocrit and hemoglobin were $22.8 \% \pm 4.5 \%$ and $7.9 \pm 1.4 \mathrm{~g} / \mathrm{dL}$, respectively. Transfused patients received $4.4 \pm 3.7$ PRBC during ICU stay and $2.2 \pm 1$ PRBC at each transfusion. The ICU $(39.8 \%$ versus $13.2 \% ; p<0.0001)$ and hospital $(48.8 \%$ versus $20.3 \% ; p<0.0001)$ mortality rates were higher in transfused patients. Mortality increased as the number of transfused PRBC increased $\left(R^{2}=0.91\right)$. In logistic regression, predictive factors for PRBC transfusion were hepatic cirrhosis, mechanical ventilation (MV), type and duration of ICU admission, and hematocrit. The independent factors associated to hospital mortality were MV, transfusions of more than five PRBC and SAPS II score.

CONCLUSIONS: PRBC transfusions are frequent in ICU patients, especially in those with medical and emergency surgical complications, longer ICU stay, and hepatic cirrhosis and in need of MV. Pre-transfusion hemoglobin levels were lower than those previously reported. In our study, PRBC transfusion was associated with increased mortality.

Key Words: Blood transfusion, Intensive Care, Prognosis, Red blood cell transfusion

\section{INTRODUÇÃO}

A anemia é freqüente em pacientes críticos e as transfusões de concentrado de hemácias (CHA) são comumente utilizadas para o seu tratamento nos Centros de Tratamento Intensivo (CTI)1. Alguns estudos referem que após três dias de internação no CTI até 95\% dos pacientes estarão anêmicos ${ }^{2,3}$. Por outro lado, $85 \%$ dos pacientes internados por mais de uma semana no CTI serão transfundidos em média com 2-3 unidades de CHA por semana, chegando até a 9,5 unidades de $\mathrm{CHA}$ durante a internação ${ }^{3}$. A causa da anemia nos pacientes internados no CTI é multifatorial, e pode-se citar as flebotomias freqüentes, a baixa produção de hemácias, as perdas sanguíneas, a hemólise, a diminuição da produção de eritropoietina e os distúrbios do metabolismo do ferro ${ }^{4,5}$. Em estudos anteriores à década de 1980, o binômio hemoglobina/hematócrito de $10 \mathrm{~g} / \mathrm{dL} / 30 \%$ era considerado um "limiar transfusional" ${ }^{2,6,7}$. Esses valores eram tidos como exigência no período pré-operatório. Entretanto, principalmente a partir da última década, os benefícios e malefícios da terapia transfusional vêm sendo reavaliados ${ }^{8-11}$.

O objetivo deste estudo foi avaliar a freqüência, as indicações, os limiares transfusionais e o prognóstico de pacientes criticamente enfermos que receberam $\mathrm{CHA}$ durante a internação no CTI.

\section{MÉTODO}

No período de abril de 2001 a julho de 2002, foi realizado um estudo prospectivo de coorte no CTI do Hospital Universitário Clementino Fraga Filho da Universidade Federal do Rio de Janeiro. O CTI conta com 12 leitos e as características do CTI já foram descritas ${ }^{12}$. O estudo foi aprovado pelo Comitê de Ética em Pesquisa da instituição, sendo dispensada a obtenção do termo de consentimento livre e esclarecido.

Através de um formulário próprio, foram coletados dados epidemiológicos, presença de doenças crônicas e agudas, a causa da admissão no CTI, sinais vitais e exames de laboratório (hemograma, bioquímica, gasometria arterial) das primeiras 24 horas da admissão no CTI. Também foram coletados os escores prognósticos APACHE II (Acute Phisiologic and Chronic Health Evaluation) ${ }^{13}$ e SAPS II (Simplified Acute Physiologic Score) ${ }^{14}$ e os escores para disfunção de múltiplos órgãos e sistemas SOFA (Sequential Organ Failure Assessment) ${ }^{15} \mathrm{e}$ MODS (Multiple Organ Dysfunction Score) ${ }^{16}$ calculados nas primeiras 24 horas de internação no CTI. Durante toda a internação no CTI, foram anotados a data, o número de unidades e justificativa para a transfusão de CHA. Também foram anotadas as evoluções no CTI e no hospital.

As variáveis categóricas foram comparadas pelos testes do Qui-quadrado (com correção de Yates quando indicado) ou Exato de Fisher e as variáveis contínuas foram avaliados pelo teste $U$ de Mann-Whitney. As variáveis categóricas foram apresentadas como percentuais e as variáveis contínuas como média \pm DP ou de mediana (intervalo interquartil 25\%-75\%). O coeficiente de correlação de Spearman foi utilizado nas correlações lineares. A regressão logística binária foi utilizada para avaliar a contribuição independente das co-variáveis na probabilidade de ser transfundido durante a internação no CTI. Para isso, as variáveis com um valor de $p<0,25$ na avaliação univariada e aquelas consideradas clinicamente relevantes entraram na 
análise multivariada. Antes da realização da análise multivariada, foram estudadas as possíveis interações entre as variáveis. Quando existiu interação, selecionou-se aquela com maior significância estatística. $\mathrm{Na}$ avaliação da discriminação, foi calculada área sob a curva Receiver Operating Characteristic (ROC) $)^{17}$. A calibração foi avaliada pelo teste do goodness-of-fit (GOF) de Hosmer-Lemeshow ${ }^{18}$. Nessas análises foram utilizados os programas Sigma Stat 3.0 (SPSS Inc., Chicago, IL, EUA) e SPSS versão 10.0 (SPSS Inc., Chicago, IL, EUA). Um valor de $p<0,05$ foi considerado como nível para estatística significativa.

\section{RESULTADOS}

Durante os 16 meses do estudo foram internados 698 pacientes, sendo $55 \%$ do sexo masculino. Duzentos e quarenta e quatro (35\%) pacientes foram transfundi-

Tabela 1 - Causas para a Transfusão de Concentrado de Hemácias (CHA).

\begin{tabular}{lc}
\hline Motivo da Transfusão de CHA & Número de Pedidos - (\%) \\
\hline Anemia & $338-(66)$ \\
Hemorragia & $116-(22,6)$ \\
Coagulação Intravascular Dis- & $34-(6,6)$ \\
seminada & \\
Outras causas & $25-(4,9)$ \\
\hline
\end{tabular}

Baseado no Motivo para a Transfusão $(n=513)$. dos durante a internação, principalmente nos primeiros quatro dias $(82,4 \%)$. As principais justificativas para a transfusão de CHA foram anemia (cerca de 2/3 dos pacientes) e hemorragias. As causas estão apresentadas na tabela 1. Os limiares transfusionais médios foram: hematócrito $=22,8 \% \pm 4,5 \%$ e hemoglobina $=7,9 \pm$ 1,4 g/dL. Em média, os pacientes receberam 4,4 $\pm 3,7$ $\mathrm{CHA}$, sendo que foram 2,2 $\pm 1 \mathrm{CHA}$ por pedido. No total, foram realizadas 513 transfusões sendo transfundidas 1056 unidades de CHA. Geralmente, os pacientes receberam dois $\mathrm{CHA}$ por transfusão.

Nas tabelas 2 e 3, são comparadas as características demográficas, diagnósticas e clínico-laboratoriais dos pacientes transfundidos e daqueles que não receberam transfusão de CHA através da estatística univariada. Trinta e quatro pacientes eram portadores de cardiopatia isquêmica. Este subgrupo de pacientes foi menos transfundido que os não portadores de cardiopatia isquêmica (transfundidos $=5 / 34-(14,7 \%)$ versus não transfundidos $=239 / 659(36,3 \%) ; p=0,01)$, mas os limiares transfusionais médios observados foram mais elevados: hematócrito $24,8 \% \pm 1,7 \%$ e hemoglobina $=8,6 \pm 0,7 \mathrm{~g} / \mathrm{dL}$.

A regressão logística selecionou cinco variáveis relacionadas à maior probabilidade de transfusão no CTI: a necessidade de VM no primeiro dia de CTI, o diagnóstico de cirrose hepática, o número de dias de internação no CTI, tipo de internação, o valor mais baixo do hematócrito também nas primeiras 24 horas de CTI e o valor

Tabela 2 - Dados Demográficos e Diagnósticos entre os Pacientes Transfundidos e os Não Transfundidos.

\begin{tabular}{|c|c|c|c|}
\hline Características $^{\star \star}$ & Pacientes Transfundidos $(n=244)$ & Pacientes não Transfundidos $(n=454)$ & Valor de $p$ \\
\hline Idade (anos) & $55(39,5-66,5)$ & $53(41-65)$ & 0,59 \\
\hline \multicolumn{4}{|l|}{ Tipos de internação } \\
\hline Clínica & $89-36,5 \%$ & $107-23,6 \%$ & $<0,001$ \\
\hline Cirurgia eletiva & $100-41 \%$ & $307-67,6 \%$ & \\
\hline Cirurgia de urgência & $55-22,5 \%$ & $40-8,8 \%$ & \\
\hline Cirrose hepática & $42-17,2 \%$ & $29-6,4 \%$ & $<0,0001$ \\
\hline Insuficiência renal crônica & $24-9,8 \%$ & $25-5,5 \%$ & $<0,05$ \\
\hline Insuficiência renal aguda & $23-9,4 \%$ & $20-4,4 \%$ & $<0,02$ \\
\hline Disritmia cardíaca & $17-7,0 \%$ & $13-2,9 \%$ & $<0,02$ \\
\hline Pneumonia à admissão & $45-18,4 \%$ & $31-6,8 \%$ & $<0,0001$ \\
\hline Sepse* & $111-45,5 \%$ & $82-18,1 \%$ & $<0,0001$ \\
\hline Ventilação mecânica* & $146-59,8 \%$ & $92-20,3 \%$ & $<0,0001$ \\
\hline Uso de vasopressores* & $80-32,8 \%$ & $62-13,7 \%$ & $<0,0001$ \\
\hline Icterícia* & $39-16,0 \%$ & $44-9,7 \%$ & 0,02 \\
\hline Necessidade de hemodiálise & $35-14,3 \%$ & $35-7,7 \%$ & 0,008 \\
\hline
\end{tabular}

* nas primeiras 24 horas de internação no CTI. ** dados categóricos apresentados como proporções (\%); dados contínuos apresentados como mediana (intervalo interquartil $25 \%-75 \%$ ) 
Tabela 3 - Dados Clínicos-Laboratoriais entre os Pacientes Transfundidos e os não Transfundidos.

\begin{tabular}{|c|c|c|c|}
\hline Características $^{\star \star}$ & Pacientes Transfundidos $(n=244)$ & Pacientes não Transfundidos $(n=454)$ & Valor de $\mathrm{p}$ \\
\hline PAS (mmHg) & $96(78,5-160,5)$ & $116(88-161)$ & 0,002 \\
\hline $\mathrm{PAD}(\mathrm{mmHg})$ & $57,5(44,5-91,0)$ & $70(52-97)$ & $<0,0001$ \\
\hline Freqüência cardíaca (bpm) & $114(72,5-129)$ & $94(63-117)$ & $<0,0001$ \\
\hline Freqüência respiratória (irpm) & $24(20-28)$ & $23(20-25)$ & 0,008 \\
\hline Diurese $(\mathrm{mL})$ & $1840(800-2919,5)$ & $2030(1347,5-3047)$ & 0,02 \\
\hline Escala de coma de Glasgow* & $15(15-15)$ & $15(15-15)$ & 0,051 \\
\hline Hematócrito (\%) & $26,2(22,1-31,7)$ & $33(28,4-37,7)$ & $<0,0001$ \\
\hline Glicose (mg/dL) & $151(110-204)$ & $137(106-184)$ & 0,03 \\
\hline Uréia (mg/dL) & $41(27-80)$ & $31(21-50)$ & $<0,0001$ \\
\hline Creatinina $(\mathrm{mg} / \mathrm{dL})$ & $1,1(0,7-1,9)$ & $0,9(0,6-1,3)$ & $<0,0001$ \\
\hline $\mathrm{PaCO}_{2}(\mathrm{mmHg})$ & $36,4(33-41,9)$ & $38(34,7-43,5)$ & 0,008 \\
\hline $\mathrm{HCO}_{3}^{-}(\mathrm{mmHg})$ & $19,9(16,2-23,7)$ & $20,8(18-24,3)$ & 0,03 \\
\hline $\mathrm{PaO}_{2}(\mathrm{mmHg})$ & $123(86,2-182,4)$ & $109,9(83,5-158)$ & 0,006 \\
\hline $\mathrm{SaO}_{2}(\%)$ & $98,1(95,4-99,4)$ & 97,7 (95-99) & $<0,04$ \\
\hline $\mathrm{FiO}_{2}(\%)$ & $40(32-70)$ & $32(21-40)$ & $<0,0001$ \\
\hline $\mathrm{PaO}_{2} / \mathrm{FiO}_{2}$ & $366,2(293,8-445,1)$ & $299,8(215,8-402,8)$ & $<0,0001$ \\
\hline APACHE II (pontos) & $17(12-23)$ & $12(8-16)$ & $<0,0001$ \\
\hline APACHE II (\%) & $23(10,2-46,9)$ & $8,5(4,3-19,1)$ & $<0,0001$ \\
\hline SAPS II (pontos) & $39(25-52)$ & $23(14-36)$ & $<0,0001$ \\
\hline SAPS II (\%) & $23(6,5-50,7)$ & $5,2(1,7-18,1)$ & $<0,0001$ \\
\hline SOFA (pontos) & $7(4-10)$ & $3(2-6)$ & $<0,0001$ \\
\hline MODS (pontos) & $5(3-8)$ & $2(1-4)$ & $<0,0001$ \\
\hline Dias de internação no CTI & $6(3-16)$ & $2(1-3)$ & $<0,0001$ \\
\hline Dias após o CTI & $7(0-16)$ & $6(3-15)$ & 0,01 \\
\hline Dias de Hospitalização & $28(14,5-47)$ & $20(9-34)$ & $<0,0001$ \\
\hline
\end{tabular}

PAS - pressão arterial sistólica; PAD - pressão arterial diastólica

${ }^{*}$ nas primeiras 24 horas de internação no CTI. ** dados contínuos apresentados como mediana (intervalo interquartil 25\%-75\%).

do escore SAPS II (Tabela 4). O modelo apresentou boa calibração pelo goodness-of-fit de Hosmer-Lemeshow e boa discriminação, avaliada pela área abaixo da curva ROC.

Os pacientes transfundidos apresentaram maior leta- lidade no CTI (transfundidos $=97 / 244-39,8 \%$ versus não transfundidos $=60 / 454-13,2 \% ; \mathrm{p}<0,0001$ ) e hospitalar (transfundidos $=119 / 244-48,8 \%$ versus não transfundidos $=92 / 454-20,3 \% ; p<0,0001)$. Quanto maior foi o número de $\mathrm{CHA}$ transfundidos, maior foi a

Tabela 4 - Análise Multivariada dos Fatores Associados com a Transfusão de CHA no CTI.

\begin{tabular}{lcc}
\hline Variáveis & RC (IC 95\%) & Valor de p \\
\hline Idade (anos) & $1,01(0,99-1,02)$ & 0,222 \\
Ventilação mecânica no primeiro dia de CTI & $3,64(1,36-5,19)$ & 0,004 \\
Tipos de internação & $2,66(1,36-5,19)$ & \\
$\quad$ Clínica & 1,00 & 0,156 \\
$\quad$ Cirurgia eletiva & $1,62(0,83-3,14)$ & $<0,001$ \\
$\quad$ Cirurgia de urgência & $3,64(1,86-7,13)$ & $<, 001$ \\
Hematócrito (unidade percentual) & $0,85(0,82-0,88)$ & 0,075 \\
Cardiopatia isquêmica & $0,26(0,06-1,15)$ & $<0,001$ \\
Cirrose hepática & $3,18(1,72-5,87)$ & $<0,001$ \\
SAPS II (pontos) & $1,07(1,05-1,09)$ & $<0,001$ \\
Duração da internação na UTI (dias) & $1,18(1,13-1,23)$ & $<$ \\
\hline
\end{tabular}

Goodness-of-fit de Hosmer-Lemeshow = 10,661; $\mathrm{p}=0,222$; Área abaixo da curva ROC = 0,89 (IC 95\% = 0,87-0,92).

$\mathrm{CHA}=$ concentrado de hemácias; CTI = centro de tratamento intensivo; RC = razão de chances; IC = intervalo de confiança; SAPS = Simplified Acute Physiology Score 
letalidade hospitalar (Figura 1). Na figura 2 estão apresentadas as curvas de sobrevida em 30 dias dos pacientes transfundidos e não transfundidos. Na análise mutivariada, três variáveis foram relacionadas ao óbito hospitalar: necessidade de VM no primeiro dia de internação no CTI, o número de CHA transfundidos e valor do escore SAPS II (Tabela 5). Para este modelo, a calibração e a discriminação também foram adequadas. A idade não foi associada de modo independente com maiores probabilidades de transfusão com $\mathrm{CHA}$ ou de óbito hospitalar.

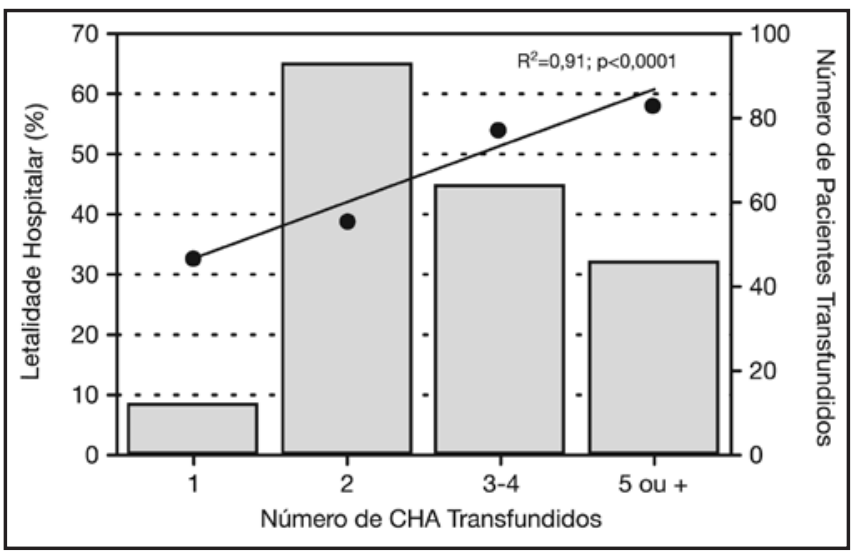

Figura 1 - Relação entre o Número de Pacientes (barras cinza), Número de CHA Transfundidos (pontos e regressão linear) e o Prognóstico Hospitalar.

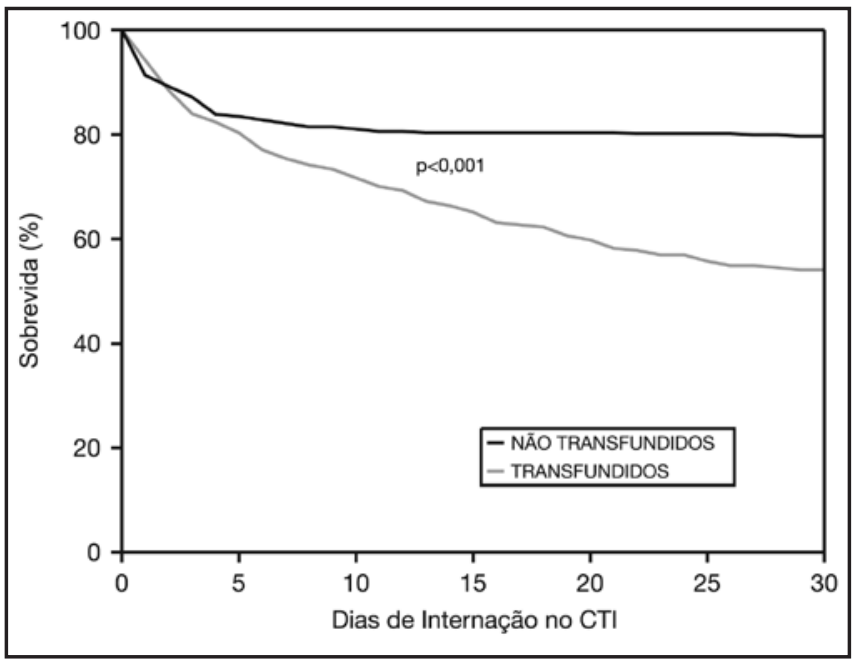

Figura 2 - Curva de Sobrevida em 30 dias de CTI dos Pacientes não Transfundidos e Transfundidos. Observou-se diferença significativa na sobrevida entre os grupos.
Tabela 5 - Análise Multivariada dos Fatores Associados com o Óbito Hospitalar

\begin{tabular}{lcc}
\hline Variáveis & $\mathrm{RC}(\mathrm{IC} 95 \%)$ & Valor de $\mathrm{p}$ \\
\hline Idade (anos) & $1,00(0,99-1,02)$ & 0,779 \\
Ventilação mecânica no primeiro & $3,69(2,08-6,55)$ & $<0,001$ \\
dia de CTI & & \\
Tipos de internação & 1,00 & \\
$\quad$ Clínica & $0,59(0,33-1,06)$ & 0,076 \\
$\quad$ Cirurgia eletiva & $0,83(0,44-1,60)$ & 0,582 \\
$\quad$ Cirurgia de urgência & & \\
Número de Transfusões (unida- & & \\
des) & 1,00 & \\
0 & $1,06(0,57-1,99)$ & 0,856 \\
$1-2$ & $1,68(0,83-3,43)$ & 0,153 \\
$3-4$ & $2,00(1,02-3,92)$ & 0,044 \\
$\geq 5$ & $1,07(1,05-1,09)$ & $<0,001$ \\
\hline SAPS II (pontos) & &
\end{tabular}

Goodness-of-fit de Hosmer-Lemeshow $=3,305 ; \mathrm{p}=0,914$; Área abaixo da curva ROC $=0,90$ IC $95 \%=0,88-0,93$.

$\mathrm{RC}=$ razão de chances; IC = intervalo de confiança; SAPS = Simplified Acute Physiology Score

\section{DISCUSSÃO}

O presente estudo é um dos poucos estudos nacionais sistematizados sobre transfusão em uma unidade de terapia intensiva clinico-cirúrgica. É importante assinalar que ele foi realizado após a publicação do estudo TRICC ${ }^{8}$, que estabeleceu novos parâmetros sobre limiares transfusionais. A comparação com outros trabalhos selecionados na literatura recente demonstra várias similaridades em relação ao tempo de internação, gravidade mensurada pelos escores APACHE ॥ e SOFA, número de transfusões por paciente, número de pacientes transfundidos e letalidade (Tabela 6). Porém, foram observadas algumas diferenças. Os limiares transfusionais médios desses estudos variaram de 8,4 a $8,6 \mathrm{~g} / \mathrm{dL}$ de hemoglobina. Em nosso estudo o limiar transfusional médio foi mais baixo $(7,9 \mathrm{~g} / \mathrm{dL}$ de hemoglobina). No estudo $\mathrm{ABC}^{9}$, a principal causa para transfusão de $\mathrm{CHA}$ foi o sangramento ativo (55,5\%); no estudo TRICC ${ }^{8}$ foi a hemorragia com $35 \%$; no estudo do Reino Unido (UK) ${ }^{11}$ também foi a hemorragia, mas em $72,2 \%$ dos pacientes; já no estudo CRIT ${ }^{10}$ foi a hemoglobina baixa em $90 \%$ dos pacientes. Neste estudo a principal causa para transfusão foi a anemia, ou seja, um valor baixo do hematócrito ou da hemoglobina. $\mathrm{O}$ motivo pelo qual se transfunde $\mathrm{CHA}$ deveria ser direcionado pela fisiologia do transporte de oxigênio $\left(\mathrm{DO}_{2}\right)$. Sabe-se que o $\mathrm{DO}_{2}$ é dependente da saturação arterial de oxigênio $\left(\mathrm{SaO}_{2}\right)$, débito cardíaco (DC) e da concen- 
Tabela 6 - Comparação do Atual Estudo (HUCFF) com Estudos Selecionados da Literatura.

\begin{tabular}{|c|c|c|c|c|c|}
\hline & $\mathrm{ABC}^{9}$ & CRIT $^{10}$ & $\mathrm{TRICC}^{8}$ & $\mathrm{UK}^{11}$ & HUCFF \\
\hline Número de pacientes estudados & 3534 & 4892 & 5298 & 1247 & 698 \\
\hline Local do estudo & Europa Ocidental & EUA & Canadá & Reino Unido & Brasil - RJ \\
\hline Período do estudo & nov/1999 & ago/00 a abr/01 & nov/94 a nov/97 & fev/99 a out/99 & $\mathrm{abr} / 01 \mathrm{a}$ jul/02 \\
\hline Hemoglobina à admissão (g/dL) & $11,3 \pm 2,3$ & $11,0 \pm 2,4$ & $9,9 \pm 2,2$ & - & - \\
\hline$\%$ de pacientes transfundidos & $37 \%$ & $44,1 \%$ & $25 \%$ & $53,4 \%$ & $35 \%$ \\
\hline$\%$ de transfusões por paciente & $4,8 \pm 5,2$ & $4,6 \pm 4,9$ & $4,6 \pm 6,7$ & $5,7 \pm 5,2$ & $4,4 \pm 3,7$ \\
\hline Número de dias de CTI & 4,5 & $7,4 \pm 7,3$ & $4,8 \pm 12,6$ & - & $6,1 \pm 10,3$ \\
\hline Letalidade no CTI (\%) & $13,5 \%$ & $13 \%$ & $22 \%$ & $21,5 \%$ & $22,5 \%$ \\
\hline Letalidade hospitalar (\%) & $20,2 \%$ & $17,6 \%$ & - & - & $30,3 \%$ \\
\hline APACHE II (pontos) & $14,8 \pm 7,9$ & $19,7 \pm 8,2$ & $18 \pm 11$ & $18,1 \pm 9,1$ & $14,9 \pm 8,6$ \\
\hline SOFA (pontos) & $5,2 \pm 3,8$ & $6,2 \pm 3,7$ & - & - & $5,2 \pm 4,3$ \\
\hline
\end{tabular}

$\mathrm{H}$ - hemorragia; $\mathrm{A}$ - anemia

tração de hemoglobina $(\mathrm{Hb})$.

$\mathrm{DO}_{2}=\mathrm{Hb}(\mathrm{g} / \mathrm{dL}) \times \mathrm{SaO}_{2}(\%) \times \mathrm{DC}(\mathrm{L} / \mathrm{min})$

A diminuição do transporte de oxigênio abaixo de níveis críticos necessários para o metabolismo oxidativo pode resultar em metabolismo anaeróbico tecidual ${ }^{19}$. Devido ao fato de que as necessidades teciduais podem estar aumentadas durante a doença aguda, é intuitiva a idéia de que a manutenção do $\mathrm{DO}_{2}$ resultaria em melhor prognóstico. Inclusive, há alguns anos, o objetivo terapêutico para pacientes gravemente enfermos era a manutenção de níveis supranormais de $\mathrm{DO}_{2}{ }^{20}$. Entretanto, estudos subseqüentes não conseguiram demonstrar melhora significativa na $\mathrm{DO}_{2}$ ou consumo de oxigênio de pacientes sépticos transfundidos com um $\mathrm{CHA}^{21}$. Porém, nesse estudo, o tempo médio de armazenamento do CHA transfundido foi de $12,8 \pm 8,1$ dias. Sabe-se que quando as hemácias são armazenadas nos banco de sangue por intervalos acima de 15 dias, a capacidade da hemoglobina para transportar oxigênio e sua deformabilidade para atravessar os capilares é essencialmente perdida ${ }^{22}$. Após duas semanas de armazenamento existe redução das concentrações de 2,3-difosfoglicerato, reduzindo o transporte de oxigênio em cerca de $50 \%{ }^{23}$. Assim, apenas os efeitos adversos da transfusão sanguínea seriam mantidos. Portanto, em pacientes gravemente enfermos deve-se evitar a transfusão de CHA com mais de 12 dias de armazenamento ${ }^{23,24}$. Em nosso hospital, devido à carência de doadores, raramente são transfundidos $\mathrm{CHA}$ com mais de uma semana de estocagem.

Em concordância com nossos dados, uma subanálise (post-hoc) do estudo CRIT com pacientes internados com trauma, a maioria $(87,1 \%)$ foram transfundidos nos primeiros quatro dias de internação, (25). Já von Ahsen e col. ${ }^{26}$ em um estudo de 99 pacientes clínicos observaram que metade das transfusões ocorreu nos primeiros cinco dias. No estudo $\mathrm{CRIT}^{10}$ cerca de $51 \%$ das transfusões foram realizadas nos primeiros três dias de internação no CTI e $65,8 \%$ dos pacientes foram transfundidos nos primeiros seis dias.

No estudo $A B C^{9}$, foi observado maior incidência de transfusão de $\mathrm{CHA}(41 \%)$ nos pacientes submetidos a cirurgias eletivas e contrariamente aos nossos achados, os pacientes clínicos transfundiram menos. Por outro lado, em concordância com nossos dados, os pacientes admitidos após cirurgia de urgência receberam mais transfusões.

No estudo $\mathrm{CRIT}^{10}$ também foi observado maior número de dias de internação no CTI e hospitalar nos pacientes transfundidos, e mais, quanto maior o número de transfusões, maior a permanência. Também se observou maior tempo de internação nos pacientes transfundidos. Por outro lado, quanto maior o tempo de internação no CTI maior a probabilidade de ser transfundido.

Em concordância com estes dados, outros estudos também observaram maior letalidade nos pacientes transfundidos $8,9,11,27$ e naqueles que receberam maior número de transfusões de $\mathrm{CHA}^{9,10}$. Entretanto, contrariamente ao estudo $A B C^{9}$, não se observou aumento do número de transfusões com a idade. Tal fato também foi constatado no estudo CRIT ${ }^{10}$.

O motivo pelo qual os pacientes transfundidos apresentam pior prognóstico permanece controverso. Muito provavelmente os pacientes mais graves são mais transfundidos, conforme demonstram os escores de 
gravidade APACHE II, SAPS II e os escores de disfunção múltipla orgânica MODS e SOFA. Alguns estudos sugerem que a exposição a antígenos leucocitários pode desencadear alterações da resposta imune acarretando maior risco de infecção e óbito ${ }^{28,29}$ e lesão pulmonar aguda induzida pela transfusão (TRALI) ${ }^{30}$. A TRALI, secundária a ativação de leucócitos, é uma das principais causas de óbito relacionadas à transfusão. Sua forma leve muitas vezes não é reconhecida pelo intensivista, fazendo com que sua verdadeira incidência seja desconhecida. Shorr e col. ${ }^{31}$ observaram que os pacientes transfundidos apresentaram maior incidência de pneumonia associada à ventilação mecânica. Por sua vez, Braga e col. ${ }^{32}$ observaram maior incidência de infecção de pacientes submetidos a cirurgia gastrintestinal por neoplasia. Existem evidências da redução da freqüência de eventos clínicos adversos com a leucorredução do $\mathrm{CHA}^{33}$. Porém, ainda não existem estudos que comparem $\mathrm{CHA}$ leucodepletados com mais e com menos de 15 dias de estocagem.

Também permanece controverso se pacientes portadores de cardiopatia isquêmica deveriam ser mais transfundidos ${ }^{34}$. Embora estudos de laboratório tenham sugerido que concentração elevada de hemoglobina seja necessária para manter uma oferta adequada de oxigênio na circulação coronariana, a própria transfusão pode causar efeitos adversos ${ }^{35}$. Em estudo retrospectivo de 1958 pacientes que não foram transfundidos por razões religiosas ${ }^{36}$, foi observado maior risco de óbito nos pacientes portadores de doença cardiovascular. Entretanto, em um estudo no qual foram comparados dois limiares transfusionais (8 e $9 \mathrm{~g} / \mathrm{dL}$ de hemoglobina) não foram observados efeitos adversos no prognóstico de pacientes submetidos a cirurgia de revascularização coronariana ${ }^{37}$. Uma subanálise (post-hoc) dos pacientes cardiopatas do estudo TRICC não mostrou efeitos deletérios no grupo restritivo ${ }^{38}$. Assim, como não existem estudos prospectivos aleatórios em pacientes cardiopatas (especialmente os portadores de isquemia miocárdica), aconselham-se condutas mais conservadoras, situando-se o limiar transfusional em cerca de $9-10 \mathrm{~g} / \mathrm{dL}^{39}$. Porém, é necessário cautela, pois a transfusão de $\mathrm{CHA}$ pode induzir edema pulmonar agudo em pacientes com doenças valvulares, ou disfunção sistólica ou diastólica de ventrículo esquerdo. Este estudo contou com poucos pacientes isquêmicos que foram menos transfundidos. Entretanto, constatou-se limiares transfusionais médios mais elevados nesses pacientes.

Os pacientes em VM receberam mais transfusão (Tabela 2). Através da regressão logística foi observado que os pacientes em prótese ventilatória apresentaram maior probabilidade de serem transfundidos (Tabela 4) e maior risco de letalidade hospitalar (Tabela 5). Provavelmente a VM entrou como um indicador da gravidade do paciente, pois foi observado que aqueles mais graves receberam $\mathrm{CHA}$ mais freqüentemente. Porém, a correção da diminuição do transporte de oxigênio utilizando transfusão de CHA não alterou o prognóstico de pacientes ventilados mecanicamente em uma subanálise (post-hoc) do estudo TRICC ${ }^{40}$. Ou seja, os pacientes que receberam uma estratégia liberal em relação à transfusão de CHA não apresentaram tempo de VM, número de dias livre do ventilador, tempo até o desmame, número de disfunções de órgãos e tempo de internação no CTI ou hospitalar diferentes. Pelo contrário, foi observada uma tendência a menor mortalidade naqueles submetidos a uma estratégia restritiva. $A$ subanálise retrospectiva do estudo $\mathrm{CRIT}^{41}$ revelou que os pacientes em VM receberam mais transfusão de $\mathrm{CHA}$ apresentaram maior letalidade e permaneceram mais tempo internados no CTI e no hospital. Concluem que a razão pela qual os pacientes em VM foram mais transfundidos deverá ser investigada.

Também foi observado que os pacientes portadores de cirrose hepática apresentaram maior probabilidade de serem transfundidos. Isso decorre da incidência elevada de hemorragia digestiva (conseqüência da hipertensão portal), seja por varizes esofagianas, seja por lesão aguda da mucosa gastrintestinal.

Em concordância com estes dados, outros estudos também demonstraram maior valor dos escores prognósticos e para disfunção de múltiplos órgãos e sistemas $^{9-11}$ em pacientes transfundidos.

Os médicos que trabalham na terapia intensiva muitas vezes utilizam um valor limite arbitrário para transfundir os pacientes anêmicos hemodinamicamente estáveis. Ao invés de se aconselhar um determinado nível, devería-se orientar a transfusão por necessidades fisiológicas definidas individualmente.

Por outro lado, podem-se diminuir as transfusões de CHA utilizando-se técnicas de cirurgia sem sangue, preparo pré-operatório (uso de eritropoietina e doação autóloga), uso de técnicas cirúrgicas e anestésicas, de máquinas para reaproveitar o sangue, (cell saver) hemodiluição peri-operatória, diminuição do desperdício da coleta de sangue com uso de tubos pediátricos e reaproveitamento de sangue retirado do cateter de pressão arterial média. Existem ainda as alternativas ao uso de hemoderivados, citando-se os carreadores artificiais do oxigênio ${ }^{42}$. 


\section{LIMITAÇÕES DO ESTUDO}

Trata-se de um estudo em um único hospital público na Cidade do Rio de Janeiro em um período de tempo determinado. A interpretação dos resultados deste estudo observacional, deve ser limitada à identificação e a descrição das variáveis de interesse. A estatística multivariada (regressão logística) mensura a associação entre as variáveis e o desfecho e não necessariamente associações causais. Além disso, o tratamento de pacientes gravemente enfermos é complexo e muitas das variáveis relacionadas não foram coletadas. Adicionalmente, não houve qualquer influência sobre os médicos do CTI em relação às suas práticas transfusionais. Sabe-se também que novas condutas e protocolos podem influenciar a prática transfusional, por exemplo o algoritmo da reanimação hídrica precoce de pacientes com choque séptico, que sugere seja mantido um valor de hematócrito acima de $30 \%{ }^{43}$. Sendo assim, é necessária cautela na extrapolação dos dados aqui apresentados para outros hospitais públicos ou privados no país.

\section{CONCLUSÃO}

Mais de um terço dos pacientes admitidos no CTI foram transfundidos, sendo geralmente administrados dois concentrados de hemácias por vez. A principal justificativa para transfusão foi a anemia, seguida da hemorragia. Os pacientes internados por problemas clínicos e aqueles admitidos em pós-operatório de urgência foram mais transfundidos. O limiar transfusional observado foi mais baixo que nos grandes estudos publicados na literatura. Os pacientes em ventilação mecânica e os portadores de cirrose hepática foram mais transfundidos. Os pacientes mais transfundidos ficaram mais tempo internados no CTI e no hospital. Além disso, os pacientes transfundidos apresentaram valores dos escores APACHE II, SAPS II, SOFA e MODS mais elevados. A quantidade de CHA transfundidos correlacionou-se com a mortalidade hospitalar.

\section{REFERÊNCIAS}

01. Napolitano LM - Scope of the problem: epidemiology of anemia and use of blood transfusions in critical care. Crit Care, 2004;8:(Suppl2):S1-S8.

02. Cane RD - Hemoglobin: how much is enough? Crit Care Med, 1990;18:1046-1047.

03. Corwin HL, Parsonnet KC, Gettinger A - RBC transfusion in the ICU. Is there a reason? Chest, 1995;108:767-771.

04. Corwin HL, Gettinger A, Rodriguez RM et al - Efficacy of recombinant human erythropoietin in the critically ill patient: a randomized, double- bind, placebo-controlled trial. Crit Care Med, 1999;27:2346-2350.

05. Rodriguez RM, Corwin HL, Gettinger A et al - Nutritional deficiencies and blunted erythropoietin response as causes of the anemia of critical illness. J Crit Care, 2001;16:36-41.

06. Adam RC, Lundy JS - Anesthesia in cases of poor risk: some suggestions for decreasing the risk. Surg Gynecol Obstet, 1942;74:1011-1101.

07. Allen JB, Allen FB - The minimum acceptable level of hemoglobin. Int Anesthesiol Clin, 1982;20:1-22.

08. Hebert PC, Wells G, Blajchman MA et al - A multicenter, randomized, controlled clinical trial of transfusion requirements in critical care. $\mathrm{N}$ Engl J Med, 1999;340:409-417.

09. Vincent JL, Baron JF, Gattinoni L et al - Anemia and blood transfusion in critically ill patients. JAMA, 2002;288:1499-1507.

10. Corwin HL, Gettinger A, Pearl RG et al - The CRIT Study: Anemia and blood transfusions in the critically ill - current clinical practice in the United States. Crit Care Med, 2004;32:39-52.

11. Rao MP, Boralessa $\mathrm{H}$, Morgan $\mathrm{C}$ et al - Blood component use in critically ill patients. Anaesthesia, 2002;57:530-534.

12. Rocco JR, Soares M, Gago MF - Pacientes clínicos referenciados, mas não internados na terapia intensiva: prevalência, características clínicas e prognóstico. RBTI, 2006;18:114-120.

13. Knaus WA, Draper EA, Wagner DP et al - APACHE II: a severity of disease classification system. Crit Care Med, 1985;13:818-829.

14. Le Gall JR, Lemeshow S, Saulnier F - A new simplified Acute Physiologic Score (SAPS II) based on a European/North American multicenter study. JAMA, 1993;270:2957-2963.

15. Vincent JL, Moreno R, Takala J et al - The SOFA (Sepsis-related Organ Failure Assessment) score to describe organ dysfunction/failure. On behalf of the Working Group on Sepsis-Related problems of the European Society of Intensive Care Medicine. Intensive Care Med, 1996;22:707710.

16. Marshall JC, Cook DJ, Christou NV et al - Multiple organ dysfunction score: a reliable descriptor of a complex clinical outcome. Crit Care Med, 1995;23:1638-1652.

17. Hanley JA, McNeil BJ - The meaning and use of the area under a receiver operating characteristic (ROC) curve. Radiology, 1982;143:29-36.

18. Lemeshow S, Hosmer DW Jr - A review of goodness of fit statistics for use in the development of logistic regression models. Am J Epidemiol, 1982;115:92-106.

19. Marshall JC - Transfusion trigger: when to transfuse? Crit Care, 2004;8(Suppl2):S31-S33.

20. Shoemaker WC, Appel PL, Kram HB et al - Prospective trial of supranormal values of survivors as therapeutic goals in high-risk surgical patients. Chest, 1988;94:1176-1186.

21. Fernandes CJ Jr, Akamine N, De Marco FV et al - Red blood cell transfusion does not increase oxygen consumption in critically ill septic patients Crit Care, 2001;5:362-367

22. Fitzgerald RD, Martin CM, Dietz GE et al - Transfusing red blood cells stored in citrate phosphate dextrose adenine-1 for 28 days fails to improve tissue oxygenation in rats. Crit Care Med, 1997;25:726-732.

23. Marik PE, Sibbald WJ - Effect of stored-blood transfusion on oxygen delivery in patients with sepsis. JAMA, 1993;269:3024-3029.

24. Weil MH - Blood transfusions. Crit Care Med, 2003;31:2397-2398.

25. Shapiro MJ, Gettinger A, Corwin HL et al - Anemia and blood transfusion in trauma patients admitted to the intensive care unit. J Trauma, 2003;55:269-274.

26. von Ahsen N, Muller C, Serke S et al - Important role of nondiagnostic blood loss blunted erythropoietic response in the anemia of medical intensive care patients. Crit Care Med, 1999;27:2630-2639.

27. Magno LA, Rezende E, Assunção $M$ et al - Transfusão sangüínea intra-operatória e mortalidade em pacientes submetidos à cirurgia eletiva para retirada de neoplasia gastrintestinal. RBTI, 2004;16:9-13.

28. Vamvakas EC, Blajchman MA - Deleterious clinical effects of transfusionassociated immunomodulation: fact or fiction. Blood, 2001;97:11801195.

29. Taylor RW, Manganaro L, O'Brien $\mathrm{J}$ et al - Impact of allogenic packed red blood cell transfusion on nosocomial infection rates in the critically ill patients. Crit Care Med, 2002;30:2249-2254.

30. Silliman CC, Ambruso DR, Boshkov LK - Transfusion-related acute lung injury Blood, 2005;105:2266-2273.

31. Shorr AF, Duh MS, Kelly KM et al - Red blood cell transfusion and ventilator-associated pneumonia: A potential link? Crit Care Med, 
2004;32:666-674.

32. Braga M, Vignali A, Radaelli G et al - Association between perioperative blood transfusion and postoperative infection in patients having elective operations for gastrointestinal cancer. Eur J Surg, 1992;158:531-536.

33. Hebert PC, Fergusson D, Blajchman MA et al - Clinical outcomes following institution of the Canadian universal leukoreduction program for red blood cell transfusions. JAMA, 2003;289:1941-1949.

34. Herbert PC, Szick S - The anemic patient in the ICU: How much does the heart tolerate? Anaesthesiol Intensivmed Notfallmed Schmerzther, 2001;36:(Suppl2):S94-S100.

35. Goodnough LT - Risks of blood transfusion. Crit Care Med, 2003;31:(Suppl):S678-S686.

36. Carson JL, Duff A, Poses RM et al - Effect of anaemia and cardiovascular disease on surgical mortality and morbidity. Lancet, 1996;348:1055-1060.

37. Bracey AW, Radovancevic R, Riggs SA et al - Lowering the hemoglobin threshold for transfusion in coronary artery bypass procedures: effect on patient outcome. Transfusion, 1999;39:1070-1077.
38. Hebert PC, Yetisir E, Martin C et al - Is a low transfusion threshold safe in critically ill patients with cardiovascular diseases? Crit Care Med, 2001;29:227-234.

39. Freudenberger RS, Carson $\mathrm{JL}$ - Is there an optimal hemoglobin value in the cardiac intensive care unit? Curr Opin Crit Care, 2003;9:356-361.

40. Hebert PC, Blajchman MA, Cook DJ et al - Do blood transfusions improve outcomes related to mechanical ventilation? Chest, 2001;119:18501857.

41. Levy MM, Abraham E, Zilberberg M et al - A descriptive evaluation of transfusion practices in patients receiving mechanical ventilation. Chest, 2005;127:928-935.

42. Goodnough LT, Shander A, Brecher ME - Transfusion medicine: looking to the future. Lancet, 2003;361:161-169.

43. Rivers E, Nguyen B, Havstad S et al - Early goal-directed therapy in the treatment of severe sepsis and septic shock. N Engl J Med, 2001;345:1368-1377. 